\title{
Leading Twist Parton Distribution Amplitudes in Heavy Fla- vor Pseudoscalar Mesons From Dyson-Schwinger Equations of QCD
}

\author{
Minghui Ding ${ }^{1,}$ a , Fei Gao ${ }^{1, b}$, Lei Chang ${ }^{2, ~ c}$, Yu-Xin Liu ${ }^{1, d}$, and Craig D. Roberts ${ }^{3, e}$ \\ ${ }^{1}$ Department of Physics and State Key Laboratory of Nuclear Physics and Technology, Peking University, \\ Beijing 100871, China \\ ${ }^{2}$ CSSM, School of Chemistry and Physics, University of Adelaide, Adelaide SA 5005, Australia \\ ${ }^{3}$ Theory Group, Physics Division, Argonne National Laboratory, 9700 S. Cass Ave., Lemont, IL 60439, USA
}

\begin{abstract}
We compute the valence-quark leading twist parton distribution amplitudes (PDAs) of heavy pseudoscalar mesons $\eta_{c}$ and $\eta_{b}$ and find that they are both broader than the $\delta$-like function while narrower than the asymptotic one. The evolution of distribution amplitude with momentum scale is then considered and PDAs will turn to a asymptotic form when the momentum goes to infinity.
\end{abstract}

\section{Introduction}

The $B_{C}$ meson had been studied under various approaches since its observation by the CDF and D0 group in different channels [1-3]. The theoretical researches include the non-relativistic QCD approach [4], QCD sum rules [5], full lattice QCD [6] approaches and so on. Among them QCD light-cone sum rules method for example, its valence-quark parton distribution amplitudes (PDAs) of c-meson or b-meson were introduced through an integration over light-cone wave functions, which were ansatz presented by Brodsky-Huang-Lepage [7]. Once the distribution amplitude was obtained, it will be widely applicable for QCD hard exclusive process. Numbers of the measurable quantities such as transition form factors and the branching ratio for particular decay channel could be computed from theory [8].

Recently pion's distribution amplitude has been obtained directly from a projection of the pion's Bethe-Salpeter wave function onto the light-cone [9], and the asymptotic form was also obtained naturally, $\phi_{\pi}^{a s y}(x)=6 x(1-x)$. The distribution amplitude of pion was broader than the asymptotic one, which explained well the emergent phenomenon of dynamic chiral symmetry breaking. In order to get the Bethe-Salpeter wave function, the quark propagator was introduced after solving QCD's Dyson-Schwinger equations (DSEs), and the bound state appeared with Bethe-Salpeter equations (BSEs) [10].

\footnotetext{
a. e-mail: mhding@pku.edu.cn, speaker

b. e-mail: hiei@pku.edu.cn

c. e-mail: lei.chiong@gmail.com

d. e-mail: yxliu@pku.edu.cn

e. e-mail: c.d.roberts@anl.gov
} 
Heavy flavor pseudoscalar meson leading twist PDAs are then planned to be studied after a clear image of pion. In the heavy flavour sector pseudoscalar mesons $\eta_{c}$ and $\eta_{b}$ are well established with the meson picture $\bar{c} c$ and $\bar{b} b$. PDAs of these two mesons are calculated in this document under the framework of DSEs following this new method that successfully applied to pion.

\section{Distribution amplitude and Bethe-Salpeter wave function}

The projection of Bethe-Salpeter wave function onto the light-cone can be written as follows

$$
f_{\eta_{c}\left(\eta_{b}\right)} \phi(x)=\operatorname{tr}_{C D} Z_{2} \int_{d k}^{\Lambda} \delta\left(n \cdot k_{+}-x n \cdot P\right) \gamma_{5} \gamma \cdot n \chi(k ; P),
$$

where the trace is over colour and spinor indices; $\int_{d k}^{\Lambda}$ is a four-dimensional integral over momentum, with $\Lambda$ the ultraviolet regularization mass-scale ; $Z_{2}(\zeta, \Lambda)$ is the quark wave function renormalisation constant, with $\zeta$ the renormalisation scale; $f_{\eta_{c}\left(\eta_{b}\right)}$ is the leptonic decay constant; $n$ is light-like fourvector, $n^{2}=0 ; P$ is the four-momentum of the meson, $P^{2}=-m_{\eta_{c}\left(\eta_{b}\right)}^{2}$ and $n \cdot P=-m_{\eta_{c}\left(\eta_{b}\right)}$, with $m$ being the meson mass; and the Bethe-Salpeter wave function

$$
\chi(k ; P)=S\left(k_{+}\right) \Gamma(k ; P) S\left(k_{-}\right),
$$

with $\Gamma$ the Bethe-Salpeter amplitude, $\mathrm{S}$ the dressed light quark propagator, and $k_{+}=k+\eta P, k_{-}=$ $k-(1-\eta) P, \eta \in[0,1]$. In detail, the dressed propagator satisfies the Gap equation

$$
S(p)^{-1}=Z_{2}\left(i \gamma \cdot p+m^{b m}\right)+Z_{1} \int_{k}^{\Lambda} g^{2} D_{\mu v}(p-k) \frac{\lambda^{a}}{2} \gamma_{\mu} S(k) \frac{\lambda^{a}}{2} \Gamma_{\nu}(k ; p),
$$

where $D_{\mu v}$ is the gluon propagator; $\Gamma_{v}$, the quark-gluon vertex; $m^{b m}$, the current-quark bare mass ; and $Z_{1}$ the quark vertex renormalisation constant.

Similarly the Bethe-Salpeter amplitude can be obtained from a homogeneous Bethe-Salpeter equation $[\Gamma(k ; P)]_{t u}=\int_{q}^{\Lambda}[S(q+) \Gamma(q ; P) S(q-)]_{s r} K_{t u}^{s r}(q, k ; P)$, in which $K$ is the full-amplitude quarkantiquark scattering kernel [11]. The axial-vector Ward-Takahashi identity enables the relation existing between the kernels in the gap equation and that in the BSE.

\section{Nakanishi-like representation}

We follow the approach introduced in Ref. [9] and use a Nakanishi-like representation of meson Bethe-Salpeter wave functions that are obtained via numerical solution of Bethe-Salpeter equation. Computation of the distribution amplitude is obtained by interpolating quark propagator and the Bethe-Salpeter amplitude separately. The $S(p)$ and $\Gamma(k ; P)$ can be expressed in the following way :

$$
S(p)=[-i \gamma \cdot p+M] \Delta_{M}\left(p^{2}\right), \quad \Gamma(k ; P)=i \gamma_{5} \frac{M^{3}}{f_{\eta_{c}\left(\eta_{b}\right)}} \int_{-1}^{1} d z \rho_{v}(z) \Delta_{M}^{v}\left(k_{+z}^{2}\right),
$$

where $\Delta_{M}(s)=1 /\left[s+M^{2}\right], \rho_{v}(z)=\frac{1}{\sqrt{\pi}} \frac{\Gamma(v+3 / 2)}{\Gamma(v+1)}\left(1-z^{2}\right)^{v}, k_{ \pm z}=k-(1 \mp z) P / 2$. Inserting Eqs. (4) into Eq. (1), using a Feynman parametrisation to combine denominators, shifting the integration variable, one can get the moments of the parton distribution amplitude $\phi(x)$. Algebraical analysis shows that different spectral function $\rho_{v}(z)$ represents different meson structure in every limit case. When $v=0$, it means a point-like particle, and if $v=1$, this is the so-called QCD's asymptotic limit.

We present the quark propagator as functions with no poles on the real $p^{2}$-axis, which is consistent with the feature of confinement [12]. The real- $p^{2}$-axis mass pole splits into pairs of complex conjugate poles. Each scalar function in the Bethe-Salpeter amplitude is described in Nakanishi-like representation following the method in Ref. [9]. 


\section{Calculated distribution amplitudes}

We use a common kernel of Dyson-Schwinger equations and Bethe-Salpeter equations, rainbowladder truncation (RL), which is widely used in DSE framework. This approximation gives most of the exact results : static properties of $\pi$ - and $\rho$ - mesons and those of the neutron and proton. It also provides predictions for experiment [13]. For the gluon sector, we use the interaction described in Ref. [14], and the infrared composition of this interaction is consistent with that determined in modern studies of QCD's gauge sector [15]. The quark mass function, $M\left(p^{2}\right)=B\left(p^{2}, \zeta^{2}\right) / A\left(p^{2}, \zeta^{2}\right)$, is independent of the renormalisation point, $\zeta$. Basic meson properties we obtain is listed in the left panel of Table 1.

Furthermore, one can use the Gegenbauer polynomials to give a complete expression to the PDA

$$
\phi(x ; \tau)=\phi^{a s y}(x)\left[1+\sum_{j=2,4, \ldots}^{\infty} a_{j}^{3 / 2}(\tau) C_{j}^{3 / 2}(2 x-1)\right],
$$

with $\left\{C_{j}^{3 / 2}(2 x-1) \mid j=0, \ldots, \infty\right\}$, which is a complete orthonormal set on $x \in[0,1]$. Coefficients of the fitting that $j \geq 10$ is not required because $a_{j}^{3 / 2} \mid j \geq 10$ decreases rapidly.

The leading twist distribution amplitudes of $\eta_{c}$ and $\eta_{b}$ together with the asymptotic case are presented in the left panel of Fig. 1. They are symmetric and much narrower than the asymptotic one but broader than $\delta$-like function, which is consistent with the trend that distribution amplitude of meson varies from light to heavy, and turn to a $\delta$-like function in the heavy quark limit.

Once the distribution amplitude is obtained, the moments of it could be calculated, where $\left\langle\xi^{n}\right\rangle=$ $\int_{0}^{1} d x(2 x-1)^{n} \phi(x)$ is the $n_{t h}$-order moment of $\phi(x)$. The $0_{t h}$-order moment $\left\langle\xi^{0}\right\rangle=\int_{0}^{1} d x \phi(x)=1$ normalises $\phi(x)$. Comparison is made in moments of the distribution amplitude with a computation using QCD sum rules [16]. Results in the right panel of Table 1 show that our $\left\langle\xi^{2}\right\rangle$ is a little larger than that of Ref. [16], and this could be explained by that our distribution is broader so that the variance of it is bigger.

Then the evolution of leading twist PDA, that is the dependence of distribution amplitude on the momentum scale $\zeta$ or, equivalently, the length-scale $\tau=1 / \zeta$, is considered. The expansion coefficient $\left\{a_{j}^{3 / 2}, j=1 \ldots, \infty\right\}$ evolve logarithmically with $\tau$, and vanish as $\tau \rightarrow 0$ [7, 17]. The evolution of PDA for $\eta_{c}$ in Eq. (5) from $\tau_{1.275} \rightarrow \tau_{100}$ is displayed in the right panel of Fig. 1. It is evident that PDA becomes broader with the increase of $\zeta$, and goes to the asymptotic form when $\zeta$ is infinity finally .

Tableau 1. Left panel : Meson basic properties (Units in GeV). Right panel : Moments of PDAs $\left\langle\xi^{n}\right\rangle$.

\begin{tabular}{llllll}
\hline & $(D w)^{3}$ & $m$ & $\zeta$ & $M$ & $f$ \\
\hline$\eta_{c}$ & 0.545 & 1.275 & 1.275 & 3.0 & 0.386 \\
$\eta_{b}$ & 0.545 & 4.28 & 4.28 & 9.0 & 0.629 \\
\hline
\end{tabular}

\begin{tabular}{l|llll}
\hline & $\eta_{c}$ & $\eta_{c}[16]$ & $\eta_{b}$ & $\eta_{b}[16]$ \\
\hline$\left\langle\xi^{2}\right\rangle$ & 0.095 & 0.073 & 0.072 & 0.067 \\
$\left\langle\xi^{4}\right\rangle$ & 0.026 & 0.014 & 0.017 & 0.011 \\
\hline
\end{tabular}

In summary, we use a Rainbow-Ladder truncation of Dyson-Schwinger equations and BetheSalpeter equations in order to get the valence-quark parton distribution amplitude of heavy pseudoscalar mesons $\eta_{c}$ and $\eta_{b}$. Bethe-Salpeter wave function is acquired by interpolating quark propagator and the Bethe-Salpeter amplitude separately. After projecting the Bethe-Salpeter wave function on the light-front, we obtain the moments of distribution amplitude numerically. Then the distribution amplitude is reconstructed through a best fitting of moments of them. The computed $\eta_{c(b)}$ 's distribution amplitude shows to be much wider than a $\delta$-like function, which is the heavy quark limit, while narrower than the asymptotic case, $\phi^{a s y}(x)=6 x(1-x)$. Evolution of distribution amplitude of $\eta_{c}$ with the momentum scale $\zeta$ is also investigated. It will become broader with the increase of $\zeta$, and 

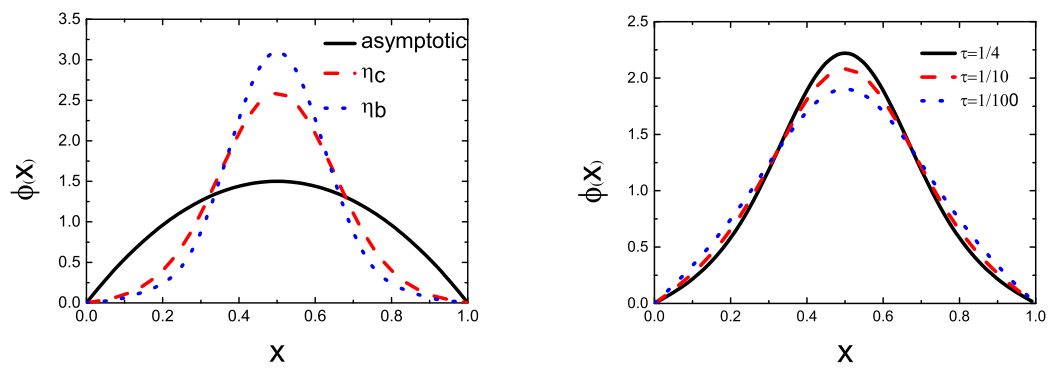

Figure 1. Left panel : Computed distribution amplitude. Curves : red dashed, PDA for $\eta_{c}$; blue dotted, PDA for $\eta_{b}$; black solid, asymptotic distribution. Right panel : Distribution amplitude evolution of $\eta_{c}$.

finally goes to asymptotic form when $\zeta$ is infinity. Study of distribution amplitude on other heavy pseudoscalar meson such as $B_{c}$ may be good verification for the variation trend we get from light meson to heavy ones, and we are in the process of it.

\section{Acknowledgement}

The work was supported by the National Natural Science Foundation of China under Contracts No. 11435001 and No. 11175004 ; the National Key Basic Research Program of China under Contract No. G2013CB834400 ; the U.S. Department of Energy, Office of Nuclear Physics, Contract No. DEAC02-06CH11357.

\section{References}

[1] F. Abe et al. (CDF Collaboration), Phys.Rev. D58, 092002 (1998)

[2] A. Abulencia et al. (CDF Collaboration), Phys.Rev.Lett. 96, 082002 (2006)

[3] M. Corcoran (CDF Collaboration, D0 Collaboration), pp. 29-32 (2005), hep-ex/0506061

[4] G.T. Bodwin, E. Braaten, G.P. Lepage, Phys.Rev. D51, 1125 (1995)

[5] V. Kiselev, A. Kovalsky, A. Likhoded, Nucl.Phys. B585, 353 (2000)

[6] E.B. Gregory, C.T. Davies, I.D. Kendall, J. Koponen et al., Phys.Rev. D83, 014506 (2011)

[7] G.P. Lepage, S.J. Brodsky, Phys.Rev. D22, 2157 (1980)

[8] M. Beneke, G. Buchalla, M. Neubert, C.T. Sachrajda, Nucl. Phys. B591, 313 (2000)

[9] L. Chang, I. Cloet, J. Cobos-Martinez, C. Roberts et al., Phys.Rev.Lett. 110, 132001 (2013)

[10] C.D. Roberts, A.G. Williams, Prog.Part.Nucl.Phys. 33, 477 (1994)

[11] P. Maris, C.D. Roberts, P.C. Tandy, Phys.Lett. B420, 267 (1998)

[12] C.D. Roberts (2012), 1203.5341

[13] P. Maris, C.D. Roberts, Int.J.Mod.Phys. E12, 297 (2003)

[14] S.x. Qin, L. Chang, Y.x. Liu, C.D. Roberts, D.J. Wilson, Phys.Rev. C84, 042202 (2011)

[15] P.O. Bowman, U.M. Heller, D.B. Leinweber, M.B. Parappilly, A.G. Williams, Phys. Rev. D70, 034509 (2004)

[16] T. Zhong, X.G. Wu, T. Huang (2014), 1408.2297

[17] A. Efremov, A. Radyushkin, Phys.Lett. B94, 245 (1980) 